\title{
Dual Self-Diffraction Dispersion-scan for Measuring Spatially Inhomogeneous Ultrashort Pulses
}

\author{
Miguel Canhota ${ }^{1, *}$, Rosa Weigand $^{2}$, and Helder Crespo ${ }^{1}$ \\ ${ }^{1}$ IFIMUP-IN and Departamento de Física e Astronomia, Faculdade de Ciências, Universidade do \\ Porto, R. do Campo Alegre 687, 4169-007 Porto, Portugal. \\ ${ }^{2}$ Departamento de Óptica, Fac. de Ciencias Físicas, Avda. Complutense s/n, Universidad \\ Complutense de Madrid, 28040, Madrid, Spain.
}

\begin{abstract}
We develop a self-diffraction dispersion-scan method that simultaneously measures two distinct spatial portions of an ultrashort light pulse and apply it to the temporal characterization of 400-nm broadband ultraviolet pulses generated by multi-plate continuum.
\end{abstract}

\section{Introduction}

Ultrafast light sources can be very complex both in the temporal and the spatial domains, and need to be correctly characterized for different applications. Among these sources, the recently developed multi-plate continuum (MPC) method [1] shows promise for solving the energy and wavelength limitations normally found in guided supercontinuum geometries, namely gas-based hollow-fiber post-compressors.

MPC uses a series of thin plates distributed along the Rayleigh range of a focused beam to generate an ultra-broadband supercontinuum, while avoiding self-focusing and filamentation within the solid plates. MPC has been demonstrated with pulses from a Ti:Sapphire amplifier [1] and with their second-harmonic in the ultraviolet [2].

An important aspect of the MPC technique is its energy scalability, since larger pulse energies can be achieved by distributing a higher energy input pulse over a larger area in the plates. However, pulsed beams tend to be less homogeneous the higher their energy, which together with the easier onset of filamentation when working with uv pulses can result in non-homogeneous spectral broadening across the MPC beam.

The characterization of such a beam requires a suitable temporal diagnostic. Dispersionscan (d-scan) [3] is a recent approach for the simultaneous measurement and compression of femtosecond laser pulses. Its setup is fully in-line and does not require beam splitting and recombination nor temporal delay of short pulses. Experimentally, it involves measuring the spectrum of a nonlinear signal, such as second-harmonic $(\mathrm{SH})$ generation $[3,4]$, third-harmonic generation [5,6], or self-diffraction [7], as a function of dispersion applied to the pulse, e.g., by using a pulse compressor. The resulting two-dimensional trace encodes information about the spectral phase of the pulse, which can be retrieved using an optimization algorithm.

In this work we introduce a non-degenerate self-diffraction d-scan implementation and demonstrate it with MPC-compressed pulses at $400 \mathrm{~nm}$.

\footnotetext{
* Corresponding author: mcanhota@,fc.up.pt
} 


\section{Experiments and results}

Pulses from a $1 \mathrm{kHz}$ Ti:Sapphire amplifier at $800 \mathrm{~nm}$ (sub-30-fs, $\sim 1 \mathrm{~mJ}$ ) are downcollimated and sent through a $200-\mu \mathrm{m}$-thick BBO crystal, generating a $\mathrm{SH}$ uv beam $(<100$ $\mu \mathrm{J}$ ) at around $400 \mathrm{~nm}$ (Fig. 1). The SH beam is filtered with a dichroic mirror and focused with a fused silica lens $(\mathrm{f}=100 \mathrm{~cm})$ onto a series of $100-\mu \mathrm{m}$-thick fused-silica plates oriented at Brewster angle, placed within the Rayleigh range of the beam. The spacing between the plates is chosen to optimize spectral broadening while avoiding optical damage to the plates. The output broadband uv pulses (Fig. 1, right) are sent to a self-diffraction dscan setup, comprising a chirped mirror (-50 fs 2 per bounce at $400 \mathrm{~nm}, 24$ bounces used) and fused silica wedge compressor.

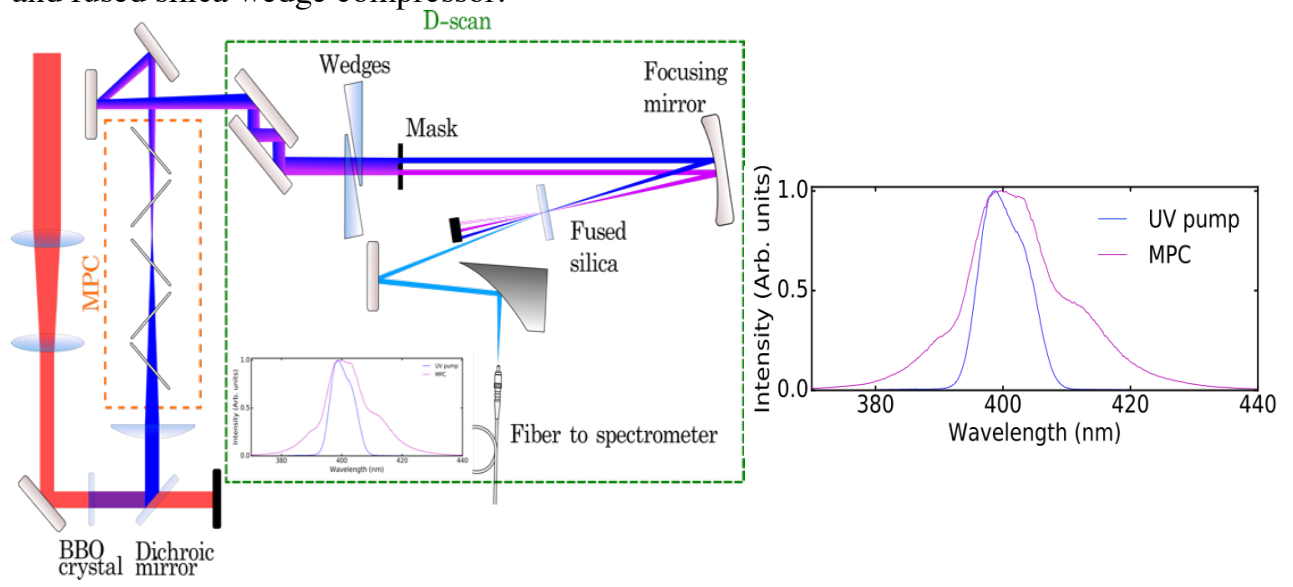

Fig. 1. (Left) Setup for generating multiplate continuum (MPC) at $400 \mathrm{~nm}$, with temporal compression using chirped mirrors and temporal characterization by dual self-diffraction d-scan. (Right) uv input and MPC output spectra.

Two different spatial portions of the beam with different spectra (dubbed Pulse 1 and Pulse 2) are then selected with a mask (two circular apertures, $1.2 \mathrm{~mm}$ diameter) and focused by a concave mirror $(\mathrm{f}=20 \mathrm{~cm})$ onto a 30 - $\mu \mathrm{m}$-thick fused-silica slide to generate non-degenerate self-diffraction signals. One of these signals is selected and its spectrum measured with a fiber-coupled spectrometer as a function of wedge insertion.

Figure 2 shows the measured trace (spectrum of the selected self-diffracted signal for different glass insertions), along with the trace retrieved by the dual self-diffraction d-scan algorithm, which is based on [7] but assumes two different fields at each aperture (with the spectra given in Fig. 3).

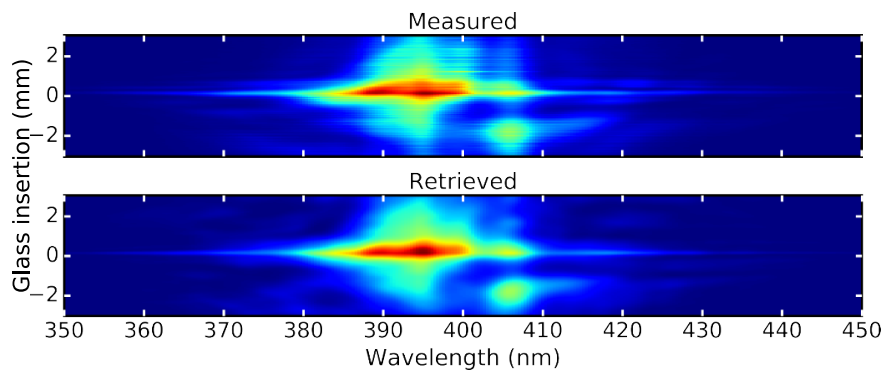

Fig. 2. Measured (top) d-scan trace and retrieved (bottom) d-scan trace assuming non-degenerate selfdiffraction. 
Figure 3 (top) shows the measured spectra and the spectral phase simultaneously retrieved for each pulse, along with their temporal intensities (bottom) compared to their transformlimited durations. We obtained compressed pulse durations of $11.6 \pm 0.1 \mathrm{fs}$ and $16.5 \pm 1.7$ fs for pulses 1 and 2, respectively.
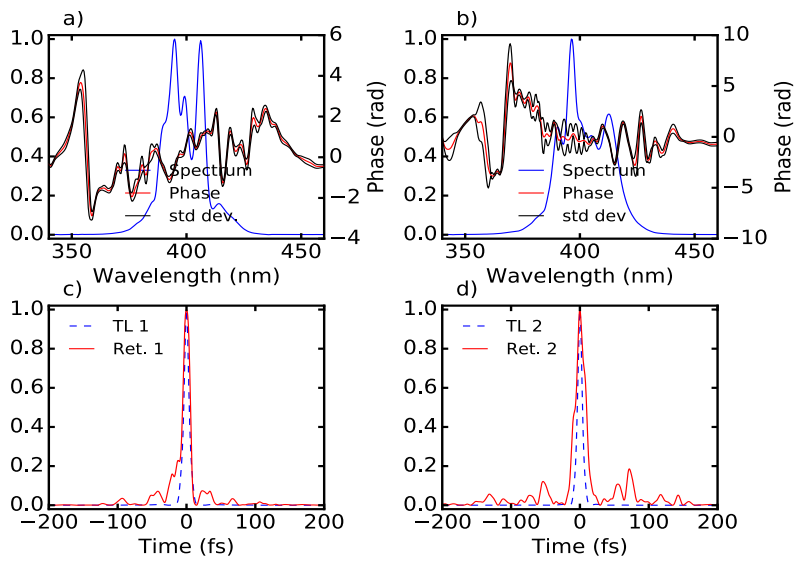

Fig. 3. (a) and (b) Spectrum and retrieved spectral phase for pulse 1 and pulse 2. (c) and (d) Pulse intensities.

Attempts to retrieve the pulses using just one spectrum (from one of the apertures) resulted in a retrieved trace less similar to the measured one (higher d-scan error) and a higher rms error in the spectral phase.

In conclusion, we have developed a method based on non-degenerate self-diffraction dscan, which is able to characterize high-intensity spatially inhomogeneous beams, and demonstrated it by simultaneously measuring the pulse temporal intensities for two different spatial portions of MPC-compressed uv pulses.

\section{References}

1. C.-H.Lu,Y.-J.Tsou,H.-Y.Chen,B.-H.Chen,Y.-C.Cheng,S.-D.Yang, M.-C. Chen, C.-C. Hsu, and A. H. Kung, Optica 1, 400 (2014).

2. Y.-Y. Liu, K. Zhao, P. He, H.-D. Huang, H. Teng, and Z.-Y. Wei, Chin. Phys. Lett. 34, 074204 (2017).

3. M. Miranda, T. Fordell, C. Arnold, A. L'Huillier, and H. Crespo, Opt. Express 20, 688 (2012).

4. M. Miranda, C. L. Arnold, T. Fordell, F. Silva, B. Alonso, R. Weigand, A. L'Huillier, and H. Crespo, Opt. Express 20, 18732 (2012).

5. F. Silva, M. Miranda, S. Teichmann, M. Baudish, M. Massicotte, F. Koppens, J. Biegert, and H. Crespo, CLEO: 2013, OSA Technical Digest (online) (Optical Society of America, 2013), paper CW1H.5.

6. M. Hoffmann, T. Nagy, Th. Willemsen, M. Jupé, D. Ristau and U. Morgner, Opt. Express 22, 5234 (2013).

7. M. Canhota, F. Silva, R. Weigand, and H. M. Crespo, Opt. Lett. 42, 3048 (2017). 